\title{
OUTCOME OF ANTIRETROVIRAL TREATMENT IN PATIENTS WITH AIDS MONOINFECTION AND THOSE CO-INFECTED WITH HEPATITIS B AND C VIRUSES AND TUBERCULOSIS IN UKRAINE
}

\author{
Olga M. Radchuk ${ }^{1,2}$, Nelly P. Chentsova ${ }^{1}$, Sergii V. Tukaiev ${ }^{3}$ \\ ${ }^{1}$ Kyiv City AIDS Centre, Kyiv, Ukraine \\ ${ }^{2}$ Institute of Communication and Health, University of Lugano, Lugano, Switzerland \\ ${ }^{3}$ Educational and Scientific Centre, Institute of Biology, National Taras Shevchenko University of Kyiv, Kyiv, Ukraine
}

\begin{abstract}
SUMMARY
Antiretroviral therapy reduces AIDS morbidity and mortality as well as decreases HIV transmission by reducing the viral load (VL). We aimed to determine the rate of immunological and virological failure that reflects antiretroviral treatment (ART) effectiveness among the patients of the Kyiv City AIDS Centre and may lead to ART switch in the future. In $59 \%$ of patients the prescribed ATR regimen was regarded as successful, in $37 \%$ as unsuccessful and $4 \%$ of patients were excluded for lacking sufficient follow-up time. Among the cases of unsuccessful ART, the largest part of patients was experiencing secondary immunological failure, on the second place was primary immunological failure. The temporal distribution of primary and secondary failures was also defined. It was shown that the number of patients with treatment failure increases steadily. Drug resistance testing is able to reduce the number of unsuccessful ART regimens, but such testing is not a part of the routine testing in Ukraine.
\end{abstract}

Key words: antiretroviral treatment, immunological failure, AIDS monoinfection, hepatitis, tuberculosis

Address for correspondence: O. Radchuk, Vidpochynku St. 11, 03115 Kyiv, Ukraine. E-mail: olgaradchuk@gmail.com

\section{INTRODUCTION}

According to the 2009 AIDS Epidemic Update (1), the number of people living with HIV worldwide reached 33.4 million in 2008, and continues to grow. Among European countries, Ukraine is experiencing especially severe and growing national epidemic. With adult HIV prevalence higher than $1.6 \%$, Ukraine has the highest infection level reported in Europe (2). By 1 January 2009, the total number of HIV-infected individuals registered in Ukraine was 91,717, among them 10,410 AIDS patients (3). In addition, such infections as tuberculosis (TB), Hepatitis B (HBV) and C (HCV) further complicate the course of disease. The prevalence of tuberculosis in Ukraine reached 47,000 cases in 2009, and the TB/HIV co-infection rate was 19 cases per 100 TB patients (4). Around 1 million people are currently infected with $\mathrm{HCV}$ in Ukraine, with the prevalence among people living with HIV of $53.3 \%$, and those infected with tuberculosis of $46.7 \%$ (5). The data about HBV distribution in Ukraine are scarce, but the prevalence reaches more than $2 \%$ of population (6). In such circumstances ART not only reduces AIDS morbidity and mortality, but it is also believed that improved treatment access and successful ART could help decrease HIV transmission by reducing the viral load (3).

However, the beneficial impact of antiretroviral therapy is considerably restricted by the ability of HIV to mutate and reproduce itself in the presence of antiretroviral drugs, which further leads to the development of drug resistance (7-9). Despite the fact that long-term ART is toxic and associated with many metabolic disorders (10), interrupting ART leads to a rapid viral rebound, attributed to the persistence of latently-infected cellular reservoirs in some cell populations, such as macrophages, monocytes and T-lymphocytes (11).

Due to high genetic variability of HIV, mutations occur in viral genome at a high rate. The resultant hybrid genomes may create drug-resistant strains that emerge and predominate in the presence of a certain drug regardless of their initial proportion in the HIV population, whereas other (sensitive) strains are suppressed. Either a single-point mutations or an accumulation of such mutations that provide a survival advantage may lead to the resistance to a specific drug or a specific drug combination, which is an increasingly recognized problem $(9,12,13)$.

Like in other resource-limited countries, clinicians do not make individualized regimen decisions when prescribing ART in Ukraine, and regimen selection is regulated by the protocols of the Ministry of Public Health guided by the WHO recommendations. It is generally accepted that inefficient ART is associated with immunological and virological failure and clinical progression of HIV infection. The efficiency of ART is characterized by measurements of the plasma VL as well as $\mathrm{CD} 4+$ blood count without resorting to the third criterion - clinical failure. It was shown (3) that VL measurement is considered to be a more sensitive indicator of treatment failure compared to clinical or immunological criteria, and immunological criteria appear to be more appropriate for the confirmation of virological failure. 


\section{MATERIALS AND METHODS}

The positive HIV status was discovered among the individuals undergoing voluntary and mandatory routine testing, testing before invasive medical interventions, and blood donors using ELISA. The population included the individuals with various socio-economic backgrounds, inclusive those with different types of risky behaviour (injection drug users, sexual workers, etc). The cohort of the studied patients comprised those receiving ART at the Kyiv City AIDS Centre after their positive HIV status had been confirmed by the presence of HIV antigen in serum using ELISA confirmatory assays. A decision about the beginning of ART was made after the detection of VL and CD4+ status of the patients. From 1998 to 2009, ART was prescribed to 1,383 patients with the diagnosis of HIV infection, but 44 individuals were excluded from the analysis because of a lack of adherence to therapy. Thus, the study population consisted of 1,339 individuals, who were part of a cohort of 4,120 HIV-infected patients under medical supervision at the Kyiv City AIDS Centre during this period, and was composed of 574 women and 765 men. 567 patients were coinfected with $\mathrm{HCV}, 16$ patients with $\mathrm{HBV}, 68$ patients with both $\mathrm{HCV}$ and $\mathrm{HBV}$ identified by either PCR or ELISA. 135 HIV-infected individuals were coinfected with tuberculosis, among them 89 patients had serologic evidence of HCV, 2 of HBV and 9 of both HBV and HCV.

According to the Recommendations for a Public Health Approach (3), immunological failure was defined as: fall of CD4+ count to pre-treatment level or below, $50 \%$ fall from on-treatment peak value, persistent CD4+ count below 100 cells $/ \mathrm{mm}^{3}$ or inability of ART to increase CD4+ cell count $>50$ cells $/ \mathrm{mm}^{3}$ during the first year of ART under the undetectable level of the plasma VL. Virological failure was defined as plasma VL level above 5,000 copies/ml or inability to achieve undetectable plasma VL level during the first year of ART $(3,14-16)$. Primary failures were defined as fall of the CD4+ count to pre-treatment level or below, persistent CD4+ count below 100 cells $/ \mathrm{mm}^{3}$ and plasma VL level above 5,000 copies $/ \mathrm{ml}$. Secondary failures were defined as a progressive decrease in the CD4+ count and an increase of $\mathrm{VL}$ in patients, who previously showed strong improvement of those indices during 1-3 years after ART had begun. We analyzed CD4+ counts and VL levels collected before ART at the start of the prescribed regimen, and during the treatment according to the Clinical Protocol (15). VL was measured every 3 months until an undetectable level was reached. Further, it was measured every 6 months provided that the clinical state of a patient was stable. VL detection was performed using real-time amplifier Abbott m2000rt (USA) and Abbott Real Time HIV-1 Assays. CD4+ count was measured during the observation period with the frequency depending on the patient's clinical state and the initial CD4+ count, at the beginning of the treatment and every 3 months during ART. The detection of the number of CD4+ cells was performed using Coulter TQ-Prep and Coulter PrepPlus2 sample preparation stations and Beckman Coulter Epics XL flow cytometer. The data was entered into the database and analyzed using SPSS 17.0.

\section{RESULTS}

In 495 patients (37\%) the ART regimen was regarded as unsuccessful due to different reasons that will be discussed below, and
59 patients (4\%) were excluded for lacking sufficient follow-up time. In 788 patients (59\%), whose median follow-up under ART was 5.3 years (ranging from 9 to 1.5 years), we observed an increase in their CD4+ counts and a decrease in their VL, equal to the WHO and Clinical Protocol of Antiretroviral Treatment (Ukraine) recommendations. Therefore, the prescribed ATR regimen was regarded as successful. Despite that, in this group we observed 4 cases $(0.5 \%)$ of temporary viral rebound with VL level $>5,000$ that disappeared without taking any measures by the next examination (during 2-3 months) and 4 cases $(0.5 \%)$ of such rebound with VL level between 1,000 and 5,000, that disappeared as well. We also observed 122 cases $(15 \%)$ of temporary CD4+ 5-25\% fall from on-treatment peak value that disappeared as well during 3-6 months. We regard these cases as a temporary decrease in adherence to ART or a short-term illness because it is not possible to determine the exact reason of such incidents now.

Among the cases of unsuccessful ART, we separated the following groups: 252 patients (51\%) experienced secondary immunological failure (deterioration of the health condition after temporary improvement), and 139 patients (28\%) - primary immunological failure (we did not observe any immunological response to ART treatment yet the plasma VL level was undetectable). In 40 patients (8\%) we observed both primary immunological and virological failures (neither immunological, nor virological response was found), and in 28 patients (6\%) we found both secondary immunological and virological failures. 25 individuals (5\%) experienced primary virological failure (absence of virological response to the treatment from the beginning) and in 11 individuals $(2 \%)$ we observed secondary virological failure (Fig. 1). Taking into account achieved results, we consider immunological failure, both primary and secondary, as the main reason of ART failure. The prevalence of immunological failure supported by undetectable level of plasma VL may be attributed to the depressed immune system due to drug abuse or asocial life-style as well as the presence of opportunistic infections. It is shown that the majority of observed HIV-infected individuals have serologic evidence of current or past exposure to HCV and, to a lesser extent, HBV. Tuberculosis is the most common op-

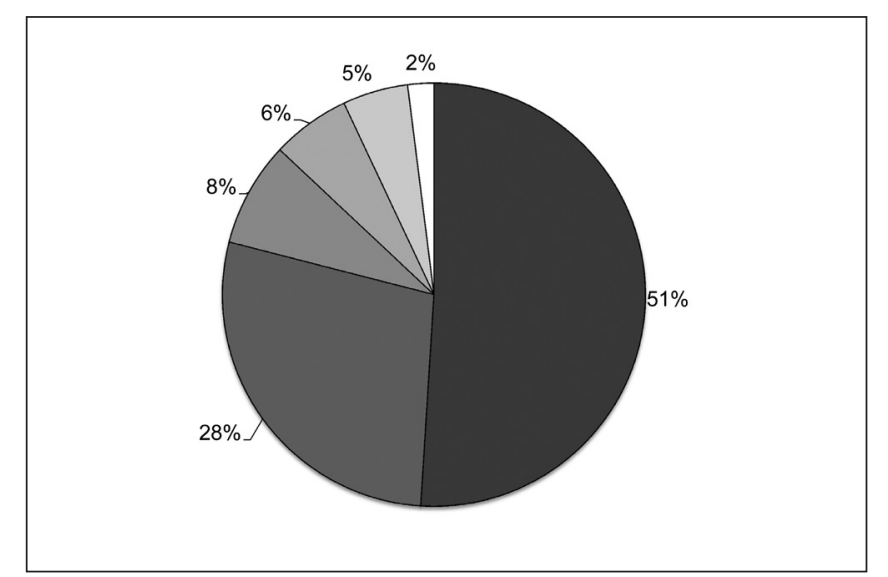

Fig. 1. Failure in ART due to:

$51 \%$ Secondary immunological failure $28 \%$ Primary immunological failure

$8 \%$ Absence of any response to the treatment

$6 \%$ Secondary immunologica and virological failure

$5 \%$ Primary virological failure

$2 \%$ Secondary virological failure 
portunistic infection affecting HIV-infected individuals, and it is the most common cause of death in patients with AIDS (2). It is well established that patients with HBV and/or HCV as well as those infected with tuberculosis and co-infected with HIV respond poorly to the therapy $(2,17)$. HIV infection accelerates the severity of chronic HCV-related liver disease, which may support further ART failure. This assumption is supported by the findings of Anderson et al. (18) and Miller et al. (19). However, the studies conducted by Yacisin et al. (20) and Antonello et al. (21) found no influence of the HCV co-infection on the progression of HIV. As such, the impact of HCV infection on the natural history of HIV and on the effectiveness of ART remains still unclear. On the contrary, the clinical manifestations of active HBV infection require an intact host immune response to mediate cell damage and destruction. Accordingly, HBV manifestation is less severe in patients with AIDS than in immunocompetent patients and they are more likely to become chronic carriers, that results in cirrhosis and more aggressive liver cell carcinoma (7, 22). Also it should be taken into consideration that antibodies to HCV may be absent in individuals coinfected with HIV, so even the patients who seem to be infected only with HIV, may be experiencing treatment failure due to HCV presence (17).

Thus, only in 57 patients (42\%) among those coinfected with $\mathrm{HIV}$ and tuberculosis/HBV/HCV their ART regimen was regarded as successful. In 71 patients (53\%) we observed primary failure (absence of any response to the treatment) and in 7 patients (5\%) we observed secondary immunological and/or virological failures. Fig. 2 represents the results of ART treatment in HIV-infected patients, coinfected with tuberculosis, HBV and HCV. This figure illustrates the prevalence of primary failures, i.e. absence of immunological and/or virological response to treatment. As such, our findings correspond to the data found in literature and confirm the problem to achieve successful and stable result of ART treatment in such patients.

It is also important to admit the temporal distribution of primary and secondary failures regardless of their nature and presence of opportunistic infections. In 2002, the rates of primary to secondary failures ranged from $16 \%$ to $84 \%$, in 2003 from $24 \%$ to $76 \%$, and in 2004 from $43 \%$ to $57 \%$. In 2005 , we observed quite significant decrease in the number of primary failures and the rate was $27 \%$ to $73 \%$. This could be due to the introduction

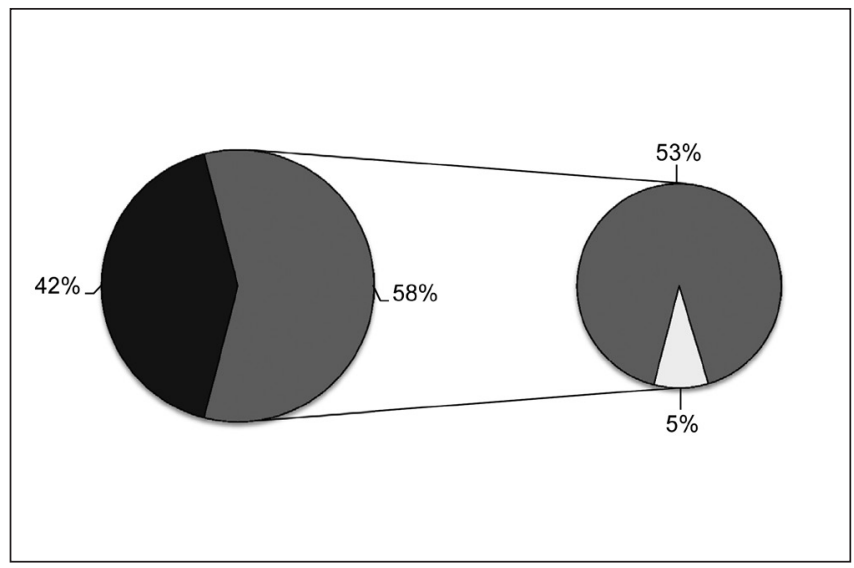

Fig. 2. ART results in HIV-infected patients, coinfected with tuberculosis, $\mathrm{HBV}$ and $\mathrm{HCV}$. of the Global Fund's programme Overcoming the HIV/AIDS Epidemic in Ukraine in 2004, which enabled broad introduction of ART treatment and expanded the variety of ART drugs, available in a triple combination. On the contrary, before 2004, the ART coverage was quite poor and in a large number of cases limited to only monotherapy. However, in 2006, the number of primary failures began to grow again and the rate ranged from $36 \%$ to $64 \%$, in 2007 from $46 \%$ to $54 \%$, in 2008 from $47 \%$ to $53 \%$, and finally in 2009 from $72 \%$ to $28 \%$, i.e. exceeded the rate of secondary failures (Fig. 3). This figure shows the strong progressive growth of primary immunological and virological failures, i.e. absence of any immunological and/or virological response from the beginning of ART. Such data may indicate the progressive increase of drug-resistant strains, circulating in Ukraine, that cause transmitted drug resistance in newly infected patients. Also it should be taken into account that in some cases such results may be observed due to poor adherence to therapy.

\section{CONCLUSIONS}

It was demonstrated that the largest part of patients among the cases of unsuccessful ART was experiencing secondary immunological failure, on the second place was primary immunological failure, and the number of patients with treatment failure increases steadily. The majority of observed HIV-infected individuals have serologic evidence of $\mathrm{HCV}$ and $\mathrm{HBV}$, and a large number of them is also co-infected with tuberculosis, that may contribute to such a poor treatment result.

Drug resistance testing is an important factor to be considered when dealing with both virological and immunological failure (9). Antiretroviral treatment and surveillance programmes have been launched in many resource-limited countries by WHO. For such programmes to be successful, it is particularly important to develop a standard list of mutations to characterize the epidemiology of the resistance type being transmitted (13). In such resource-limited settings that the ART field is experiencing in Ukraine, it is crucially important to avoid unnecessary switching to more expensive next-line regimen that may take place after the detection of immunological failure. In such cases, especially accompanied with undetectable VL levels, ART switching re-

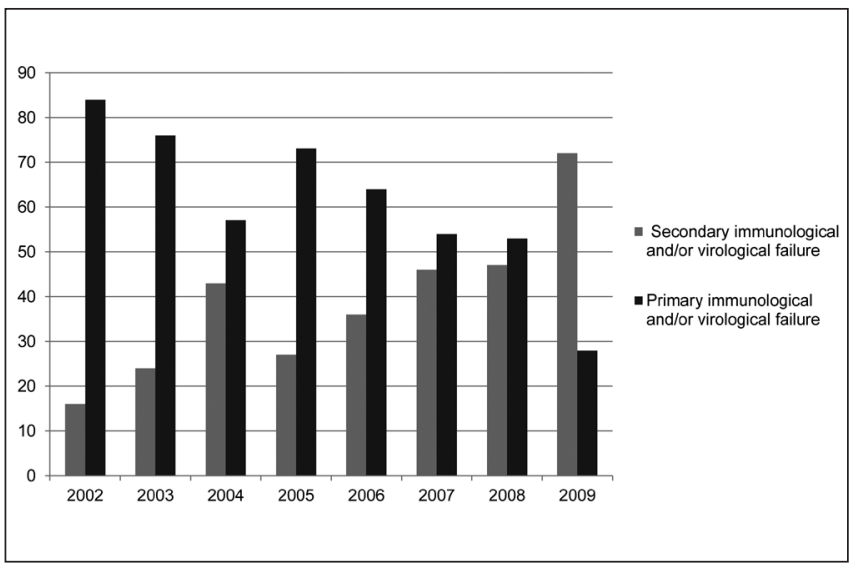

Fig. 3. Ratio of Secondary immunological and/or virological failure to Primary immunological and/or virological failure during 2002-2009. 
mains debatable. Switching ART based only on immunological and clinical evidence of a lack of ART effect may be associated also with accumulation of mutations and reduce the activity of the second-line drugs (14). In patients with CD4+ counts $<200$ cells $/ \mathrm{mm}^{3}$ the regimen may be corrected according to the present clinical manifestations by adding one more medication to suppress possible replication. It might be also recommended to switch the ART regimen to another one that includes protease inhibitor $(14,15)$.

Nowadays, it is impossible to avoid the acquired drug resistance, which develops during ART, but it is quite feasible to reduce the number of primary unsuccessful regimens by testing for drug resistance. As more antiretroviral drugs are approved, resistance profiles within drug classes have become increasingly important, so resistance testing is widely accepted in high-income countries. Unfortunately, such testing is experiencing only the initial stage of its development in Ukraine and is not being performed routinely because of lack of equipment and funding. Frozen plasma samples, obtained from newly infected patients a) before the beginning of ART, b) after 6 months of treatment (only in case of failure) and c) after 1 year (in case of failure as well) is planned to be tested outside Ukraine according to the WHO recommendations (23). The coverage of resistance testing will be extremely low - only 105 individuals (21\%) are included in the programme in contrast to $390(79 \%)$ of those in need. While these figures represent relative progress in dealing with different kinds of treatment failure, it is recognized that the current coverage and possibilities to extend it are still inadequate to exert a decisive impact on ART treatment management in general.

\section{Conflict of Interests}

None declared

\section{Acknowledgements}

We acknowledge the cooperation of the physicians of the Kyiv City AIDS Centre and Anti-AIDS Centre Laboratory for providing the data for this study.

\section{REFERENCES}

1. Joint United Nations Programme on HIV/AIDS; World Health Organization. AIDS epidemic update: December 2009. Geneva: UNAIDS; 2009.

2. Kruglov YV, Kobyshcha YV, Salyuk T, Varetska O, Shakarishvili A, Saldanha VP. The most severe HIV epidemic in Europe: Ukraine's national HIV prevalence estimates for 2007. Sex Transm Infect. 2008 Aug;84 Suppl 1:i37-i41.

3. World Health Organization. Antiretroviral therapy for HIV infection in adults and adolescents: recommendations for a public health approach: 2010 revision. Geneva: WHO; 2010.

4. MEASURE Evaluation. MEASURE Evaluation in Ukraine [Internet]. Chapel Hill: MEASURE Evaluation [cited 2014 Aug 6]. Available from: http://www.cpc.unc.edu/measure/countries/ukraine/measure-evaluationin-ukraine.
5. Ignorance is no longer an option: why and how to respond to the Hepatitis $\mathrm{C}$ epidemic in Ukraine [Internet]. Open Society Institute; 2010 [cited $2014 \mathrm{Feb} 10]$. Available from http://www.harm-reduction.org/files/pdf/4country/43en_Ukraine.pdf.

6. Centers for Disease Control and Prevention. Listing of countries with Hepatitis B prevalence $\geq 2 \%$ [Internet]. Atlanta: CDC; 2005 [cited 2014 Aug 6]. Available from: http://www.cdc.gov/ncidod/hepatitis/b/country_listing.htm\#europe.

7. Clavel C. Mechanisms of HIV drug resistance: a primer. PRN Notebook [Internet]. 2004 Mar [cited 2014 Aug 6];9(1):3-7. Available from: http:// www.prn.org/images/pdfs/277_clavel_francois.pdf.

8. Paredes R, Clotet B. Clinical management of HIV-1 resistance. Antiviral Res. 2010 Jan;85(1):245-65.

9. Hirsch MS, Günthard HF, Schapiro JM, Brun-Vézinet F, Clotet B, Hammer SM, et al. Antiretroviral drug resistance testing in adult HIV-1 infection: 2008 recommendations of an International AIDS Society-USA panel. Clin Infect Dis. 2008 Jul 15;47(2):266-85.

10. Forsythe S, Stover J, Bollinger L. The past, present and future of HIV, AIDS and resource allocation. BMC Public Health. 2009 Nov 18;9 Suppl 1:S4. doi: 10.1186/1471-2458-9-S1-S4.

11. Colin L, Van Lint C. Molecular control of HIV-1 postintegration latency: implications for the development of new therapeutic strategies. Retrovirology. 2009 Dec 4;6:111. doi: 10.1186/1742-4690-6-111.

12. Kuritzkes DR. HIV resistance: frequency, testing, mechanisms. Top HIV Med. 2007 Nov-Dec;15(5):150-4.

13. Shafer RW, Rhee SY, Pillay D, Miller V, Sandstrom P, Schapiro JM, et al. HIV-1 protease and reverse transcriptase mutations for drug resistance surveillance. AIDS. 2007 Jan 11;21(2):215-23.

14. ART failure and strategies for switching ART regimens in the WHO European Region: report of the WHO expert consultation, Copenhagen, 7 December 2007. Copenhagen: WHO Regional Office for Europe; 2008.

15. Ministry of Health of Ukraine. Clinical protocol of ARV therapy for HIV-infected adults and adolescents. Kiev: Ministry of Health of Ukraine; 2010. (In Ukrainian.)

16. Eramova I, Matic S, Munz M, editors. HIV/AIDS treatment and care: clinical protocols for the WHO European Region. Copenhagen: WHO; 2007.

17. Bilgin M, Balci NC, Erdogan A, Momtahen AJ, Alkaade S, Rau WS. Hepatobiliary and pancreatic MRI and MRCP findings in patients with HIV infection. AJR Am J Roentgenol. 2008 Jul;191(1):228-32.

18. Anderson KB, Guest JL, Rimland D. Hepatitis C virus coinfection increases mortality in HIV-infected patients in the highly active antiretroviral therapy era: data from the HIV Atlanta VA Cohort Study. Clin Infect Dis. 2004 Nov 15;39(10):1507-13.

19. Miller MF, Haley C, Koziel MJ, Rowley CF. Impact of hepatitis C virus on immune restoration in HIV-infected patients who start highly active antiretroviral therapy: a meta-analysis. Clin Infect Dis. 2005 Sep 1;41(5):713-20.

20. Yacisin K, Maida I, Ríos MJ, Soriano V, Núñez M. Hepatitis C virus coinfection does not affect CD4 restoration in HIV-infected patients after initiation of antiretroviral therapy. AIDS Res Hum Retroviruses. 2008 Jul;24(7):935-40.

21. Antonello VS, Appel-da-Silva MC, Kliemann DA, Santos BR, Tovo CV. Immune restoration in human immunodeficiency virus and hepatitis $\mathrm{C}$ virus coinfected patients after highly active antiretroviral therapy. Braz J Infect Dis. 2013 Sep-Oct; 17(5):551-4.

22. Ukraine: national report on monitoring progress towards the UNGASS Declaration of Commitment on HIV/AIDS. Reporting period: January 2006 - December 2007. Kyiv: Ministry of Health of Ukraine; 2008.

23. Bennett DE, Bertagnolio S, Sutherland D, Gilks CF. The World Health Organization's global strategy for prevention and assessment of HIV drug resistance. Antivir Ther. 2008;13 Suppl 2:1-13. 